\title{
Le rôle déterminant de la presse dans la vie des expulsés allemands
}

\author{
Lionel Picard ${ }^{1}$
}

\begin{abstract}
[Résumé] Cet article s'intéresse à l'identité des Allemands expulsés à la fin de la Seconde Guerre mondiale, par le biais de l'étude de l'un de leurs journaux, le Grafschafter Bote. Pour cette population désormais éparpillée géographiquement, les journaux publiés par les nombreuses associations participent à l'émergence puis au maintien d'une identité collective, capable de s'affirmer dans le nouvel État allemand. Ces journaux qui existent toujours contribuent ainsi à renforcer une identité particulière. Mots-clés : presse, Allemagne, expulsés, identité, histoire, mémoire.
\end{abstract}

[Abstract] This article focuses on the identity of the German expellees after World War II through the study of one of their publications, the Grafschafter Bote. For this population who is spread geographically all over Germany, the newspapers published by numerous associations have contributed to the creation and the maintaining of a collective identity able to sustain its position in the new German state. These newspapers still exist today and contribute to the strengthening of a particular identity.

Keywords: press, Germany, expellees, identity, history, memory.

\section{Introduction}

À la fin de la Seconde Guerre mondiale, plus de 14 millions d'Allemands doivent quitter la région où ils habitent, soit qu'ils fuient devant l'avancée de l'Armée rouge, soit qu'ils sont expulsés par les autorités communistes qui s'établissent à l'est de la ligne Oder-Neisse. Ils espèrent que cette migration forcée sera provisoire, et beaucoup s'engagent pendant des années pour revendiquer leur droit au retour. C'est notamment la publication de journaux qui va leur permettre de rester en lien les uns avec les autres et d'organiser les activités militantes au sein d'associations réparties dans tout le pays. La presse des expulsés poursuit des objectifs très clairs : elle veut non seulement donner des informations à ses lecteurs, mais elle se met aussi à leur service dans leur combat pour satisfaire leurs revendications. Malgré l'éloignement, elle entend participer à la reconstitution de la communauté villageoise et atténuer la souffrance liée à la perte de la Heimat ${ }^{2}$.

1 Université de Bourgogne. Centre Interlangues - Texte, Image, Langage.

2 Afin d'éviter les malentendus dans la traduction, on conserve le mot allemand "Heimat » pour désigner le lieu d'où l'on est originaire et où l'on se sent chez soi. 
Au moment de leur arrivée dans leur région d'accueil, les expulsés se trouvent dans un état de grande détresse. L'événement historique de l'expulsion a changé radicalement le cours de leur vie et ils deviennent, y compris d'un point de vue légal, des expulsés. En commençant par retracer l'évolution de la presse des expulsés, l'analyse montre, à travers une étude de cas et l'exploitation des archives d'un grand journal silésien, que la presse n'a pas seulement accompagné les expulsés, mais qu'elle a également joué un rôle essentiel dans la construction de l'identité du groupe.

\section{L'histoire de la presse des expulsés}

\subsection{Conditions de la création des journaux}

L'histoire de la presse des expulsés débute dans les mois qui suivent l'expulsion de 1945 à 1947. Arrivés dans les zones d'occupation des Alliés, les expulsés sont séparés les uns des autres. L'expulsion participe au chaos de l'après-guerre. Comme une grande partie du peuple allemand, les expulsés attendent des nouvelles de leurs proches. Les premiers journaux qui leur sont destinés prennent la forme de feuilles volantes ou de feuilles paroissiales distribuées à la sortie de l'église. Sous couvert de religion, les publications s'adressent en fait exclusivement aux expulsés (Stanzel 1955). La dimension religieuse permet en effet aux publications d'échapper à la censure des Alliés qui refusent que les expulsés se regroupent ou se structurent en associations. Cette censure nourrit les frustrations et les antagonismes en donnant aux expulsés l'impression qu'ils sont indésirables dans leur région d'arrivée et sont les mal-aimés de la nation allemande.

Le premier recensement de la presse des expulsés s'effectue dès 1953. Les journaux d'expulsés sont alors considérés, par les historiens et les sociologues, comme des documents de travail. Le tableau d'ensemble de la presse des expulsés est le suivant: en décembre 1952, 320 périodiques paraissent au moins quatre fois par an, dont 234 ont pour public-cible des expulsés d'une origine géographique précise. Les journaux des Allemands de Silésie et des Sudètes constituent, conjointement, la moitié de toutes ces publications (Kurth 1953). Durant cette phase de mise en place, beaucoup de ces journaux s'inscrivent dans la continuité de ceux qui existaient déjà dans la Heimat et ils en reprennent les formes (titre, mise en page, numérotation). Signe de l'attachement des expulsés à leur Heimat, ce sont les journaux concernant une région précise qui rencontrent le plus de succès, tandis que les journaux qui s'adressent à l'ensemble des expulsés sont confrontés à des difficultés économiques bien plus nombreuses : «Plus on s'adresse directement à lui et plus le lien du lecteur à son journal est fort ${ }^{3}$.» (Neu-

3 Toutes les traductions de l'allemand sont de l'auteur. 
hoff 1970) La diversité des journaux est renforcée par le fait que les éditeurs des publications n'ont pas tous le même statut. Tandis que certains journaux sont les organes de presse officiels de grandes organisations comme la Fédération des expulsés (Bund der Vertriebenen) ou les différentes associations nationales d'expulsés d'une même région (Landsmannschaften), la plupart sont des publications d'organisations régionales, de groupes locaux ou encore des bulletins paroissiaux. Ces différents niveaux d'organisation des médias reflètent en partie les identités multiples que développent les expulsés, partagés entre ancienne et nouvelle Heimat.

En 1963, un pasteur expulsé de Breslau/Wrocław entreprend de dresser l'inventaire complet des publications consacrées à la Silésie et brosse le tableau du paysage médiatique de la région, de ses origines, au XVIIIe siècle, jusqu'au début de la guerre (Bunzel). Il souhaite ainsi perpétuer la tradition journalistique silésienne. L'impression qui domine est celle d'une activité en perte de vitesse. Le pasteur constate néanmoins que les lecteurs demeurent nombreux, puisque l'ensemble des journaux rassemble alors 350000 lecteurs, ce qui correspond au tiers de tous les lecteurs de journaux en Silésie, en 1935.

Des années plus tard, en 1970, Neuhoff utilise cet inventaire de la presse pour analyser l'évolution du secteur sur une période de quinze ans. Selon lui, le groupe que constituent les expulsés allemands est, de tous les groupes de réfugiés du monde, celui dont la presse est la plus développée. Il observe une diminution du nombre de titres depuis le milieu des années 1950 mais explique ce phénomène moins par une désaffection des lecteurs que par une réorganisation du secteur marquée par la fusion de titres. Le tirage total de ces journaux est évalué à 1,75 million d'exemplaires par an, soit une baisse d'environ $12 \%$ de 1957 à 1969. Le mitan des années 1950 correspond à l'apogée de la presse des expulsés, qu'il s'agisse des titres publiés ou des exemplaires vendus. Malgré tout, Neuhoff conclut à une surprenante stabilité du secteur car les lecteurs ne se détournent pas de leur journal et lui restent fidèles à travers le temps. Effectivement, des dizaines de journaux d'expulsés continuent de paraître chaque mois, au début du XXIe siècle.

\subsection{Contenu des journaux}

L'étude qu'Isolde Stanzel a menée en 1955 constitue la tentative la plus élaborée d'établir une typologie de la presse des expulsés. Son travail témoigne de la difficulté à classifier ces publications. Elle propose quatre grandes catégories: les journaux ( Zeitungen »), les revues ( Zeitschriften »), les lettres de la Heimat (« Heimatbriefe ») et les lettres d'informations et service de presse («Mitteilungsblätter» / « Pressedienste »). Ces quatre catégories sont ensuite à nouveaux divisées en quatre sous-catégories, car les publications initialement prises en compte ne sont pas toutes considérées comme de « vrais » journaux. Pour être considérée comme un « vrai » journal par Isolde Stanzel, une publication doit couvrir des sujets ne touchant pas uniquement les expulsés. 
Toutefois, ce système de classification s'avère inopérant pour certaines publications dont le caractère hybride résiste à toute tentative de systématisation. Si les classements proposés ont le mérite d'apporter un peu d'ordre et de lisibilité dans un ensemble peu cohérent a priori, aucun ne permet de réellement catégoriser la presse des expulsés. La dimension artisanale des conditions de production ainsi que les changements fréquents à la tête des rédactions font de ces journaux des objets d'étude difficilement saisissables.

Bunzel établit aussi en 1963 une liste des caractéristiques de la presse des expulsés. Un certain nombre d'entre elles participent de la construction de l'identité des expulsés :

Les journaux parlent d'abord du souvenir de la Heimat ;

Ils montrent un grand nombre de photos ;

Le dialecte de la Heimat est utilisé, entre autres dans des chansons et poèmes :

Une grande place est accordée à l'histoire (pas seulement récente) ;

Le souvenir de l'effondrement de 1945 est aussi entretenu ;

Les nouvelles des familles expliquent en grande partie le succès de la diffusion des journaux ;

Les publicités servent à financer le journal et à soutenir des magasins qui entretiennent la tradition silésienne.

Il est frappant de constater que ces caractéristiques datant du début des années 1960 sont encore valables pour les journaux actuels. La continuité du contenu est un trait marquant de la presse des expulsés, comme si les journaux étaient figés dans le temps comme l'est restée la Heimat dans l'esprit des expulsés.

À cette même époque, les tirages élevés des journaux témoignent de leur succès auprès des expulsés. Parmi les 3,25 millions d'Allemands expulsés de Haute-Silésie et Basse-Silésie quinze ans plus tôt, près de 350000 lisent l'un de ces journaux. Ces publications répondent initialement à un besoin de se rassembler émanant d'individus isolés. Les rubriques « carnet », « nouvelles des familles » ou " avis de recherche » dans les années d'après-guerre en témoignent. En rassemblant les expulsés autour de valeurs et de revendications communes, ces journaux comblent un vide et contribuent à la naissance d'une identité de groupe.

\section{2. Émergence d'une identité : l'exemple du Grafschafter Bote (GB)}

\subsection{Qu'est-ce que le GB?}

Le GB, le Grafschafter Bote, paraît depuis le 15 janvier 1950 chaque mois sans interruption. En plus de soixante ans d'existence, son équipe de rédaction n'a pas fondamentalement changé. Les deux cofondateurs, qui sont aussi les principaux rédacteurs, sont 
Georg Goebel et Alois Bartsch, tous deux originaires du Comté de Glatz (Silésie). Georg Goebel est le dernier prêtre du village de Rosenthal. Jusqu'à sa mort, en 1965, il se consacre au soutien moral et politique des expulsés. Il est l'un des plus actifs représentants des expulsés, durant les années 1950. Alois Bartsch, Georg Goebel et Peter Großpietsch, rédacteur en chef depuis 1995, sont sans conteste les rédacteurs qui ont le plus marqué le journal et influencé son évolution. Comme ils sont également extrêmement impliqués dans l'organisation de la vie politique et sociale des expulsés au sein des associations provinciales entre autres, leurs contributions renforcent le crédit de ce journal, représentatif de la presse des expulsés. Les lecteurs savent que ces rédacteurs ont acquis, hors de la rédaction, une telle légitimité que leurs articles n'expriment pas uniquement leur avis personnel, mais reflètent en partie la ligne défendue par l'ensemble des représentants des expulsés. Présents dans les médias plus généralistes et utilisant ces relais pour donner de l'ampleur aux idées qu'ils défendent, ils peuvent être considérés comme des faiseurs d'opinion (Schenk 2000). Les prises de position régulières des rédacteurs avant chaque élection législative sont un signe clair de ce statut. Le GB constitue entre autres l'instrument au service de ces faiseurs d'opinion à destination de la masse des récepteurs constituée par un grand nombre de lecteurs. Les idées diffusées dans le GB circulent d'autant mieux à travers le journal que celui-ci remporte un succès important auprès de son lectorat. Les expulsés achètent en effet volontiers la publication car ils savent qu'ils seront davantage confortés dans leurs idées que lors de discussions avec des personnes n’appartenant pas au réseau que forment les expulsés. A travers le $G B$, les faiseurs d'opinion contribuent à renforcer une identité homogène parmi les expulsés.

Mis sur le marché en 1950, le GB voit son tirage atteindre rapidement les 13000 exemplaires mensuels (1952). Il se stabilise à ce niveau jusqu'à la fin des années 1980. Il connaît alors une chute importante et ne compte plus que 7600 abonnés en 1984 avant d'enregistrer une hausse spectaculaire lors de l'unification en 1989. Le journal gagne environ 1500 nouveaux abonnés en quelques mois et revient à quelque 10000 abonnés. En 2011, entre 7000 et 8000 personnes reçoivent chaque mois le $G B^{4}$.

\subsection{Les objectifs du $G B$ selon sa rédaction}

Plusieurs fois, au cours de son existence, le $G B$ s'est exprimé sur les raisons de son existence afin que ses lecteurs saisissent bien la singularité du journal et, partant, ce qui le rend indispensable. Dans le premier numéro, Alois Bartsch s'adresse à ses compatriotes du Comté de Glatz et leur explique le sens de la parution du GB. Il fait le constat de la nécessité de rassembler les expulsés pour éviter qu’ils ne disparaissent

4 Interview de Peter Großpietsch par l'auteur à Lüdenscheid le 2 mai 2010. 
en s'assimilant au reste de la population allemande, et propose d'utiliser le journal pour leur faire prendre conscience de leur identité particulière :

Nous avons un devoir envers notre Heimat. La maintenir dans nos pensées, la reconquérir ou la reconstruire, cela ne nous est possible qu'en faisant front commun et en resserrant les rangs. [...] Pour toutes ces raisons, la Zentralstelle der Grafschafter et son journal d'information le Grafschafter Bote sont absolument indispensables. C'est pourquoi il a sa place dans toutes les familles de Grafschafter ${ }^{5}$ (Bartsch 1950/1).

Le $G B$ a, dès ses origines, été mis au service de ses lecteurs. La Heimat est présentée comme un idéal que tous les expulsés ont pour devoir moral de servir. En défiant les partis politiques accusés de mal représenter les expulsés et en s'opposant au reste de la population allemande jugée indifférente, le $G B$ rassemble les expulsés autour de son projet politique et il entend s'appuyer sur ce rassemblement des victimes de l'expulsion pour faire entendre leur voix qui a besoin d'un porte-parole.

Dans le deuxième numéro, la rédaction s'adresse une nouvelle fois à ses lecteurs pour rappeler son ambition, tant du point de vue éditorial que concernant sa relation aux lecteurs :

« Nous rendrons compte au fur et à mesure des événements économiques et politiques qui intéressent nos compatriotes et nous prendrons position sur les choses qui nous concernent en tant que groupe ethnique. Mais pour pouvoir en toute situation travailler et vous conseiller, votre collaboration est indispensable. » (Bartsch 1950/2)

La volonté de placer le contenu et les lecteurs du journal dans une relation étroite est manifeste.

En 2000, le cinquantenaire de la création du GB donne à la rédaction l'occasion de publier un ouvrage documentaire exceptionnel retraçant l'histoire du journal. La page de titre reproduit ce qui pourrait être un résumé de la ligne éditoriale du journal :

1950 - 2000. 50 ans du « Grafschafter Bote ». Une documentation. Au service de la Silésie :

Cohésion des Grafschafter

Maintien des traditions

Promotion des études scientifiques, historiques et civilisationnelles sur le Comté de Glatz

Défense des intérêts politiques des Silésiens expulsés, en particulier des Grafschafter (Großpietsch 2000).

L'ouvrage reflète bien l'idée que la rédaction actuelle du journal se fait du rôle du $G B$. Elle reprend les objectifs initiaux des fondateurs du journal en affichant comme

5 C'est ainsi que se désignent les habitants du Comté de Glatz. On garde le nom allemand pour éviter la lourdeur d'une périphrase en français. 
ambition le rassemblement de la communauté des Grafschafter ainsi que le désir de promouvoir la culture de la Heimat sous toutes ses formes. Elle y ajoute une dimension politique qui s'est développée à mesure que se dessinait un clivage autour de la question des expulsés à la fin des années 1960 L'ancrage actuel du journal très à droite de l'échiquier politique donne au $G B$ une identité forte qui correspond aux besoins de ses lecteurs de s'afficher dans la sphère publique.

Le GB ne s'adresse qu'à un public restreint et utilise sa défiance vis-à-vis des grands médias pour affirmer son identité propre. Le $G B$ a tendance à considérer que les médias de masse suivent une ligne éditoriale unique, proche $\mathrm{du}$ "politiquement correct», et qu'ils ignorent systématiquement les expulsés. En faisant de la question de l'expulsion le prisme à travers lequel toute l'action politique est envisagée, le $G B$ adopte un agenda setting qui lui est propre (McCombs 1972). Ce faisant, il entend prouver à ses lecteurs que l'actualité peut être envisagée sous un angle très différent de celui choisi par la plupart des journaux. Il est aujourd'hui couramment admis que les journaux ne disent plus à leurs lecteurs ce qu'ils doivent penser mais plutôt ce à quoi ils doivent penser (McCombs 2000). Dans cette perspective, le GB choisit de privilégier des événements que d'autres journaux négligent et se situe dès lors souvent en porte-à-faux par rapport aux autres publications. De manière générale, les différents médias qui circulent au sein d'une société donnent une image assez précise de ce qu'elle considère comme important (Pöttker 2005). L'opposition entre le GB et les médias dominants reflète l'antagonisme entre la vision de la société adoptée par les expulsés, et celle adoptée par le reste de la population allemande. Cependant, la presse des expulsés, aussi diverse et multiple soit-elle, ne parvient pas à concurrencer les publications de masse et n'atteint pas le « grand public » (Kittel 2007).

\subsection{Les lecteurs du $G B$ et leur attachement au journal}

Contrairement à la presse généraliste, la presse des expulsés ne s'adresse pas à l'ensemble de la population mais vise un public très particulier. De plus, tous les expulsés ne sont pas susceptibles de s'intéresser à l'ensemble des journaux qui leur sont destinés. Ainsi, les expulsés du Comté de Glatz sont répartis sur tout le territoire allemand à leur arrivée, dans l'immédiat après-guerre. Certains sont isolés et perdent le contact avec le reste de leur communauté. Le morcellement géographique des expulsés explique que seul un nombre très limité de personnes soit susceptible de lire le GB. Des années après sa création, le journal mène une campagne de promotion et tente de sensibiliser ses lecteurs. Le GB prétend maintenir le lien entre les personnes dispersées à travers le pays. Pour cela, affirme la publication, il importe que chacun des expulsés ait au moins connaissance de l'existence du journal. Le GB compte sur ses lecteurs pour assurer sa promotion auprès d'autres expulsés, et ainsi fidéliser de nouveaux lecteurs. La solidarité entre Grafschafter apparaît aussi dans le fait qu'une famille envoie le journal à une autre famille après l'avoir lu. Cette pratique s'est ins- 
tallée durant les années 1950 alors que tout le monde n'avait pas les moyens de s'offrir un abonnement personnel au journal. Cette habitude perdure et s'observe également avec d'autres journaux. Une étude réalisée en 1968 sur l'Ostpreußenblatt, un autre journal d'expulsés de Prusse orientale, montre qu'environ deux tiers des lecteurs transmettent leur journal après l'avoir lu $u^{6}$. Aujourd'hui encore, Peter Großpietsch estime qu'un exemplaire est lu dans trois familles. Cette affirmation repose sur le constat que les nouvelles publications de la maison d'édition du GB donnent lieu à trois vagues successives de commandes : la première lorsque le $G B$ arrive chez les abonnés, puis une deuxième et une troisième lorsque des parents ou amis reçoivent le numéro après lecture dans le foyer précédent? $7 e$ nombre de lecteurs est une donnée importante pour évaluer l'importance du journal chez les expulsés du Comté de Glatz, mais il ne suffit pas à rendre compte de son audience réelle. Il faut tenir compte également de son taux de circulation (Agnès 2008).

Toutefois, le lectorat du GB est limité par nature. Ses lecteurs potentiels forment un réseau uni par leur origine géographique et leur destin commun d'après-guerre. Ces deux éléments contribuent à forger un sentiment d'appartenance. Les discussions à l'intérieur de ce cercle peuvent porter sur des sujets politiques dans la mesure où c'est cette dimension qui est à l'origine de la constitution du groupe et qui reste d'actualité avec l'évolution des relations germano-polonaises. Ce sentiment d'appartenance à une communauté de destin contribue à l'émergence, dans le journal, d'une « communauté discursive ", c'est-à-dire que c'est avec les mêmes mots que parlent tous les contributeurs du journal qui appartiennent à une même communauté (Moirand 2007). De plus, les liens d'amitié qu'entretiennent de nombreux membres du groupe sont de nature à favoriser les débats politiques puisque l'on discute plus facilement de politique avec les gens dont on se sent le plus proche à l'intérieur d'un réseau (Schenk 1994). Cette congruence d'intérêts fait du journal l'instrument idéal pour façonner l'identité collective des expulsés.

Les lecteurs sont très présents dans le journal. Ils apparaissent dans la rubrique traditionnelle du courrier des lecteurs. Cette rubrique est restée très vivante depuis la création du GB, et certaines lettres ont parfois provoqué des polémiques qui ont enrichi les autres rubriques dans les mois suivant leur publication. Les « Nouvelles des familles » constituent l'autre rubrique du journal au sein de laquelle tous les lecteurs se retrouvent. À travers la mention de la date de leur anniversaire, les lecteurs voient leur nom apparaître au moins une fois dans l'année dans leur journal. De cette manière, la rubrique contribue à resserrer le lien d'attachement des lecteurs à leur jour-

6 Les résultats varient selon la profession et l'âge des lecteurs. Le journal est transmis à une personne (27\% des cas), deux personnes (34\%) ou trois personnes ou plus (39\%) (Gaida 1973).

7 Interview de Peter Großpietsch à Lüdenscheid le 2 mai 2010 par l'auteur. 
nal, ainsi qu'à renforcer la communauté en informant sur les événements de la vie des autres membres. Dans ces pages, l'identité du journal se confond avec celle de ses lecteurs. L'existence de cette rubrique n'est rendue possible que par le travail bénévole de ceux qui sont chargés de collecter et de mettre à jour les données personnelles de chacun des anciens habitants d'un village dont ils ont la charge. Enfin, le journal compte beaucoup sur ses lecteurs pour qu'ils enrichissent son contenu. C'est surtout dans les comptes rendus de voyages ou de rencontres que ces derniers sont mis à contribution. À travers leurs articles et leurs photos, les expulsés sont nombreux à se retrouver ou à voir le nom ou le visage de personnes connues. Le lien entre le lecteur et son journal est renforcé par cette participation de tous à l'élaboration du journal. Le $G B$ est un journal qui est fait par et pour ses lecteurs. Ceux-ci sont à la fois les récepteurs et les producteurs du contenu du journal.

\section{Conclusion : les journaux façonnent et reflètent simultanément l'identité des expulsés}

Depuis la fin des années 1940, pour une population plongée dans la plus grande détresse, la presse a constitué un soutien considérable. Elle a fait défaut lors de l'arrivée des expulsés dans leur nouvel environnement, car l'urgence était ailleurs. Lorsque la presse des expulsés a été autorisée, les titres se sont multipliés, preuve que ces publications répondaient à un besoin réel. Elles ont permis aux expulsés de trouver leur nouvelle identité et elles les ont accompagnés tout au long de leur intégration en République fédérale d'Allemagne. S'il est à première vue étonnant que nombre de ces journaux continuent d'exister au XXIe siècle, on découvre l'importance du secours qu'ils ont apporté aux expulsés à travers les récits et les photos proposés depuis toujours à leurs lecteurs. L'élargissement du contenu à une dimension politique que l'on observe dans certains journaux conforte les expulsés fidèles à leur publication dans une identité particulière. La presse fait alors office de refuge pour des personnes dont le traumatisme passé est si grand que le repli sur soi ou à l'intérieur du groupe apparaît comme la seule issue possible.

\section{Bibliographie}

AgNES Y. (2008) : Manuel de journalisme. Paris : La Découverte, p. 47-49.

BARTSCH A. (1950/1) : « Liebe Grafschafter Landsleute! ». In Grafschafter Bote, n 1, p. 4.

BARTSCH A. (1950/2) : «Liebe Grafschafter Landsleute! ». In Grafschafter Bote, n² 2, p. 4.

BUNZEL U. (1963) : Schlesien lebt. Zeitungen, Zeitschriften und Rundbriefe der schlesischen Heimatvertriebenen. Wurtzbourg : Holzner Verlag. 
GAIDA H.-J. (1973) : Die offiziellen Organe der ostdeutschen Landsmannschaften. Ein Beitrag zur Publizistik der Heimatvertriebenen in Deutschland. Berlin : Duncker \& Humblot.

GROßPIETSCH P. (2000): "Liebe Grafschafter und Freunde der Grafschaft Glatz in Schlesien! ». In Grafschafter Bote, $\mathrm{n}^{\circ}$ 1, p. 1-2.

KITTEL M. (2007) : Vertreibung der Vertriebenen? Der historische deutsche Osten in der Erinnerungskultur der Bundesrepublik (1961-1982). Munich: Oldenbourg Verlag, p. 40.

KuRTH K. O. (1953) (éd.) : Handbuch der Presse der Heimatvertriebenen. Kintzingen-Main : Holzner Verlag.

Mccombs M., Shaw D. (1972) : "The agenda-setting function of the mass media ». In Public Opinion Quaterly, vol. XXXVI, $\mathrm{n}^{\circ} 2$.

Mccombs M. (2000): «Agenda-Setting: Zusammenhänge zwischen Massenmedien und Weltbild », p. 123-137. In SCHORR A. (éd.) : Publikums- und Wirkungsforschung. Wiesbaden : Westdeutscher Verlag.

MoIRAND S. (2007) : Les discours de la presse quotidienne. Observer, analyser, comprendre. Paris : puf, p. 133.

Neuhoff H. (1970) : « Die Presse der deutschen Vertriebenen Anfang 1970 », p. 153-175. In A.W.R. - Bulletin. Vierteljahresschrift für Flüchtlingsfragen, $\mathrm{n}^{\circ}$ 3-4.

PÖTtKer H. (2005): "Öffentlichkeit/Öffentliche Meinung », p. 329-333. In WEISCHENBERG S., KLEINSTEUBER H. J., PÖRSKEN B. (éd.): Handbuch Journalismus und Medien. Constance : UVK.

SCHENK M. (1994) : « Meinungsbildung im Alltag - Zum Einfluß von Meinungsführern und sozialen Netzwerken », p. 143-158. In JÄCKEL M., WINTERHOFF-SPURK P. (éd.) : Politik und Medien. Analysen zur Entwicklung der politischen Kommunikation. Berlin : Vistas.

SCHENK M. (2000) : «Schlüsselkonzepte der Medienwirkungsforschung », p. 71-85. In ScHORR A. (éd.), Publikums- und Wirkungsforschung. Wiesbaden: Westdeutscher Verlag.

StANZEL I. (1955) : « Die Presse der Vertriebenen », p. 17-25. In Der neue Ackermann, n 2. 